\title{
Similarity of the perimeters in the Ebbinghaus illusion
}

\author{
JESSE M. CHOPLIN and DOUGLAS L. MEDIN \\ Northwestern University, Evanston, Illinois
}

\begin{abstract}
Coren and Miller (1974) and Coren and Enns (1993) argued that the magnitude of the Ebbinghaus illusion is a function of the rated or conceptual similarity of the inducing objects to the test object. In three experiments, we examined the convergence between conceptual similarity and illusion magnitude. The first failed to find support for this parallel. Two further experiments yielded support for an alternative hypothesis that the magnitude of the Ebbinghaus illusion is a function of the similarity of the perimeters of the inducing object to the test object. The similarity of the centers had no effect. These results suggest that the information used to estimate size is computed earlier in the visual system than suggested by Coren and colleagues and apparently does not involve the use of conceptual information.
\end{abstract}

Under the Ebbinghaus illusion, an object appears larger when surrounded by small objects than when surrounded by big objects. An example of this illusion is shown in Figure 1. The center circle (the test object) appears larger when surrounded by small circles (small inducing objects) than when surrounded by big circles (big inducing objects). Another example of this illusion can be seen in the book Gulliver's Travels: Gulliver looks big when surrounded by the Lilliputians, but he looks small when surrounded by the Brobdingnagians.

Recently, there has been renewed interest in this illusion because of work by Stanley Coren and his associates suggesting a link between the magnitude of this illusion and figural (Coren \& Miller, 1974) and conceptual (Coren \& Enns, 1993) similarity. In order to get an intuitive sense of why similarity ought to influence the magnitude of this illusion, consider an example, cited by Stanley Coren, in which a 5-ft 10-in. sports announcer appears small when surrounded by 7 - $\mathrm{ft}$ basketball players but does not appear small when standing next to the stadium. The sports announcer is similar to the basketball players but is not similar to the stadium. Because of their similarity to the sports announcer, the basketball players can serve as a standard of reference from which to judge the sports announcer's size. However, the stadium cannot serve as such a standard of reference. Therefore, there will be greater size contrast effects in similar things, such as the sports announcer and the basketball players, than in dissimilar things, such as the sports announcer and the stadium.

This work was supported by Grant BNS 95-11757 from the National Science Foundation. The authors thank Stanley Coren, Jim Enns, and two anonymous reviewers for reading an earlier version of this manuscript. The authors also thank John Hummel, their expert vision consultant, for his constant support and helpful comments throughout this project. Correspondence should be addressed to J. M. Choplin, Department of Psychology, University of California, 405 Hilgard Ave., Los Angeles, CA 90095-1563 (e-mail: choplin@psych.ucla.edu).
To study this phenomenon, Coren and Miller (1974) manipulated the figural similarity of the inducing objects to the test object and found that this manipulation had an effect on the magnitude of the size contrast. They used a circle as the test object and surrounded this circle with random angular shaped inducers in one condition, triangles in another, hexagons in yet another, and other circles in a final condition. The random angular shape was rated by participants to be significantly less similar to the test circle than the triangle, which in turn was rated less similar than the hexagon, which was rated less similar than an identical circle. As predicted, the magnitude of the Ebbinghaus illusion was directly proportional to the rated similarity of the inducing objects to the test object.

Coren and Enns (1993) extended these findings by manipulating the conceptual similarity of the inducing objects to the test object and found that conceptual similarity also affects the magnitude of size contrast. For example, for one set of stimuli, they used a test dog, inducing shoes, inducing horses, and inducing dogs of different breeds from the test dog. The test dog is conceptually less similar to the inducing shoes than it is to the inducing horses, since the test dog and the horses are domesticated animals. The inducing horses, in turn, are conceptually less similar to the test dog than are the inducing dogs. In another set of stimuli, they used a test young girl's face, inducing trucks, inducing grown men's faces, and other inducing young girls' faces. The test young girl's face is conceptually less similar to the inducing trucks than it is to the inducing grown men's faces, since both the test young girl's face and the inducing grown men's faces are types of human faces. The inducing grown men's faces, in turn, are conceptually less similar to the test young girl's face than are the inducing young girls' faces. For both sets of stimuli, the magnitude of the Ebbinghaus illusion was directly proportional to the conceptual similarity of the inducing objects to the test object. This finding was especially interesting, because it suggests that semantic information plays a role in what has generally been considered to be a lower level perceptual illusion. 


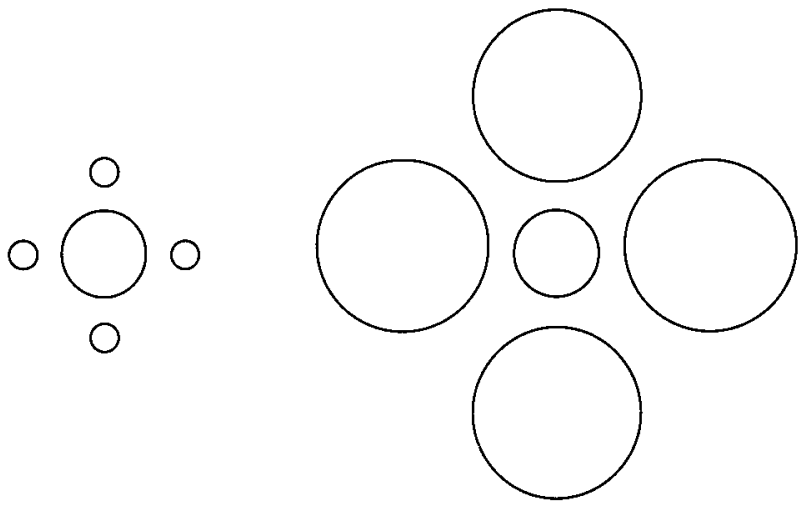

Figure 1. An example of the Ebbinghaus illusion.

In yet another experiment, they used a single design for both the test object and inducing objects. In order to manipulate the category membership of the test and inducing objects, they simply changed the orientation of the surrounding stimuli (see Figure 2). The design became a magician with a hat from one orientation and a rabbit coming out of a magician's hat from another orientation. Note that the same figures are used in the different contexts so that any illusion differences cannot be explained in terms of low-level figural similarity. The results again supported their hypothesis. When the test object was in the orientation so as to be a rabbit, the magnitude of the Ebbinghaus illusion was greater when the inducing objects were also in the orientation so as to be rabbits than when they were in the orientation so as to be magicians. Similarly, when the test object was in the orientation so as to be a magician, the magnitude of the Ebbinghaus illusion was greater when the inducing objects were also in the orientation so as to be magicians than when they were in the orientation so as to be rabbits. In a final experiment, they used schematized stimuli in an attempt to show that the change in orientation could not have produced these effects. We will discuss the efficacy of this manipulation in the General Discussion section below.

Does semantic information play the role in this illusion that Coren and Enns's (1993) results seem to imply? Or is this illusion a lower level perceptual illusion, as it has generally been held to be? We were attracted by the possibility that "size contrast may be used as a tool to study semantic organization in various populations, including college undergraduates, bilingual, brain-damaged, and developing subjecis" (Coren \& Enns, 1993, p. 587). In short, the magnitude of the Ebbinghaus illusion may provide an indirect measure of conceptual similarity. Such an indirect measure might avoid certain demand characteristics associated with more explicit similarity judgment tasks. Our initial goal, therefore, was to bolster the claims of Coren and his associates with a conceptual
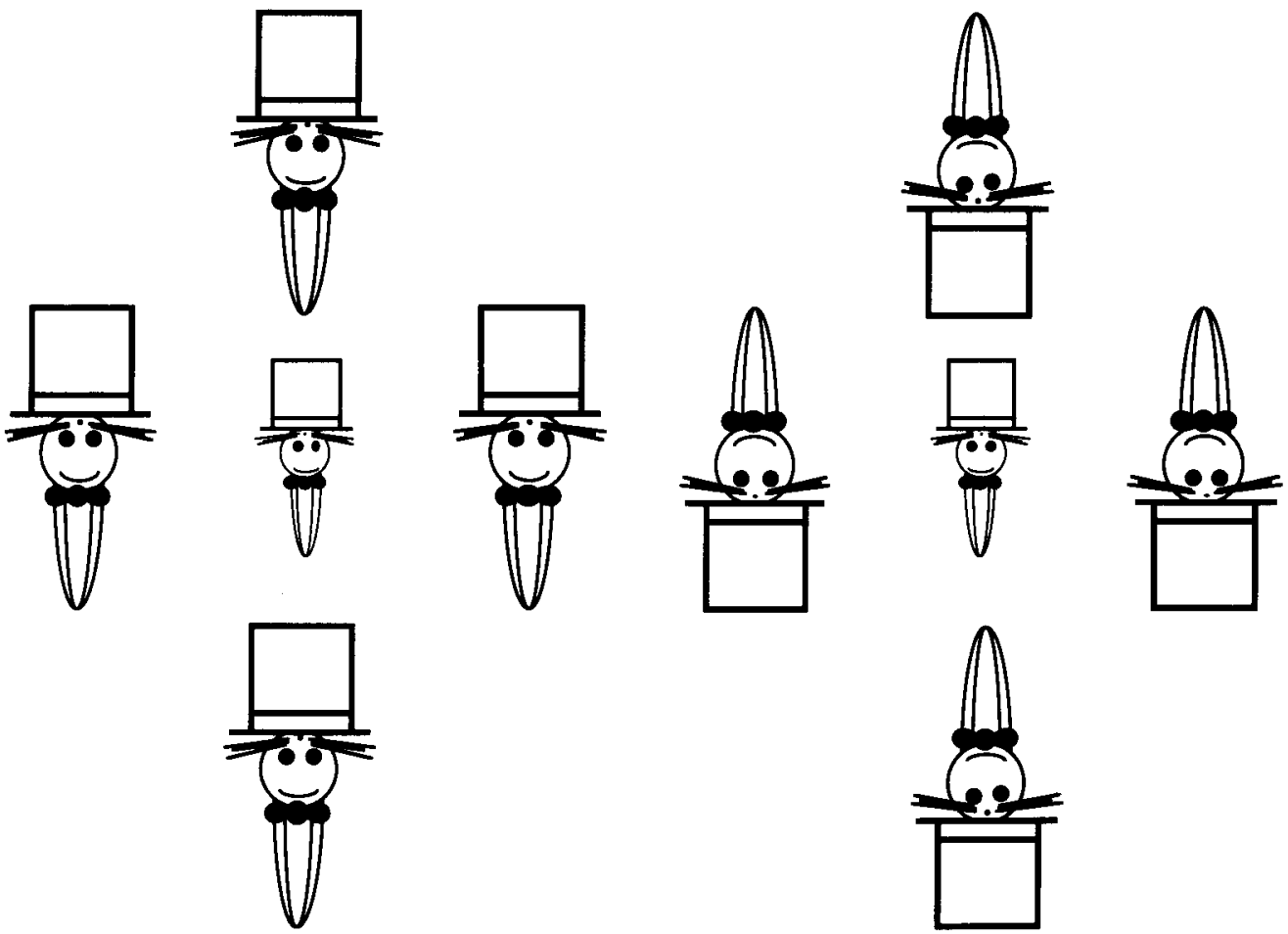

Figure 2. Stimuli used by Coren and Enns (1993) to control for the effects of figural similarity. Although the inducing objects on the left are identical to the inducing objects on the right except for orientation, the orientation of the objects on the left makes them look like magicians, whereas the orientation of the objects on the right makes them look like rabbits. 
replication. As a first step toward this goal, we picked a similarity phenomenon already established in the literature and tried to demonstrate corresponding differences in illusion magnitude.

Specifically, our focus was on the role of attributional versus relational matches in similarity (Goldstone, Medin, \& Gentner, 1991). Attributes apply to a single entity (i.e., they are one-place predicates), whereas relations link multiple entities (i.e., they are multiple-place predicates). For example, the topmost object in Figure 3 shares the one-place predicate white (left triangle) with the object to its immediate lower right. Consequently, it has an attributional match (AM) with that object. In contrast, the topmost object in Figure 3 shares the two-place predicate same-color (left triangle, right triangle) with the object immediately below it. Consequently, it has a relational match (RM) with that object.

Goldstone et al. (1991) demonstrated that RMs are more important to similarity judgments in certain contexts than are AMs. On the basis of the results of that study, we predicted that inducing objects that were identical to the test object would produce a greater size contrast effect than would inducing objects with an RM to the test object; these RM inducing objects in turn would produce a greater size contrast effect than would inducing objects with an AM to the test object.

\section{EXPERIMENT 1}

\section{Method}

Participants. The participants were randomly approached by the experimenter on the elevated trains around the Chicago area. Fiftyseven people agreed to be in the experiment after being approached in this manner. Of these 57 participants, 5 were dropped from analysis, because the experimenter determined that they had not understood the task, leaving 52 participants for analysis. All participants served voluntarily.

Materials. We designed three sets of stimuli as shown in Figure 3. Each set contained a test object and three inducing objects. The inducing objects labeled ID were identical to their test objects. The inducing objects labeled RM had a relational match with their test objects; that is, they shared the predicate same-color (left triangle, right triangle), same-shape (upper shape, lower shape), or sameshape (left shape, right shape). Finally, the inducing objects labeled AM had an attributional match with their test objects; that is, they shared the predicate white (left triangle), square (upper shape), or circle (right shape).

With the exception of four practice items at the beginning of the sequence, we designed each figure (test object with big or small inducers) such that the maximum horizontal extent across the test object was $22 \mathrm{~mm}$. To create the inducing objects, we started with designs that had the same dimensions as the test object and then scaled the dimensions of the small inducing objects down to $5 / 11$ of their original size and scaled the dimensions of the big inducing objects up to $20 / 11$ of their original size. The test objects in the practice figures varied from $10 \mathrm{~mm}$ across to $30 \mathrm{~mm}$ across at their widest extent. This variance allowed the experimenter to monitor how well the participant understood the task and to correct any misunderstanding before the actual experiment began. To control for any possible effects of distance between the test object and the induc- ers, this distance was kept constant throughout all conditions of each stimuli set. All of these figures were drawn 1 per page on a Macintosh computer and printed on a laser printer.

There were 40 items in all. Four of these items were practice items placed at the beginning of the sequence. To control for sequence effects, filler items were added, and all remaining items were block randomized. These 36 remaining items consisted of 18 experimental items - three sets of stimuli times three types of similarity relationships (ID, RM, and AM) times two (big and small inducers)-and 18 filler items.

For our dependent measure, we used the method of reproduction (Coren \& Girgus, 1972) in which participants estimate the distance across the test object at its widest extent by making a mark across a line so that the distance from a stop mark on one end of that line to the mark they make is equal to their estimate of the distance across the test object. A 40-page booklet was prepared for this purpose, 1 page per estimate that the participant was to make. Each page of this booklet was approximately $5 \mathrm{~cm}$ high and $21.6 \mathrm{~cm}$ wide. A horizontal line $14 \mathrm{~cm}$ long was provided with a vertical stop $1 \mathrm{~cm}$ high on the left-hand side.

Procedure. The participants were tested individually. The stimuli were held up for the participant to see at a distance that was constant across conditions, approximately $1 \mathrm{~m}$ away. The experimenter demonstrated the task on the first practice test object allowing the participant to observe what he or she was to do. The experimenter then asked the participant to estimate the distance across the remaining three practice test objects, during which time the experimenter monitored the participant's responses to make sure he or she understood the task. The participant then went on to estimate the distance across the remaining 36 experimental and filler test objects.

To control for serial position, each participant started at a different place in the sequence. The 1st participant started on the first item, the 2 nd participant started on the second item and did first item last, and so on. This process continued until the 37th participant who started on the first item, thus starting the process all over again.

\section{Results}

We got a measure of the magnitude of the Ebbinghaus illusion by subtracting the participant's estimate of the distance across the test object when it is surrounded by big objects from when it was surrounded by small objects. As can be seen in Table 1, the mean magnitude of the illusion was greater than zero in all nine cases, significantly greater than zero in seven of those nine.

However, a pooled analysis of variance (ANOVA) failed to reveal an effect of similarity type on the magnitude of the illusion $[F(2,106)=1.77, p>.10]$. Even more importantly, a planned comparison failed to reveal a greater magnitude illusion when the test objects were surrounded by ID inducers, which were identical to them, than when they were surrounded by RM and AM inducers, which were different $[F(1,51)=1.67, p>.10]$.

\section{Discussion}

We failed to observe any effect of similarity in this experiment. The RM and AM inducing objects produced just as great a size contrast effect as the ID inducing objects. After completing Experiment 1, we discovered that we were not the first researchers who had failed to find an effect of similarity on the magnitude of this illusion. Jaeger and Grasso (1993) surrounded test circles with 


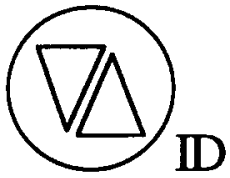

white (left triangle) white (right triangle) same-color (left triangle, right triangle)

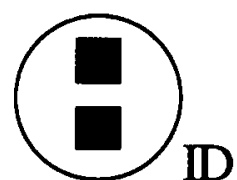

square (upper shape) square (lower shape) same-shape (upper shape, lower shape)

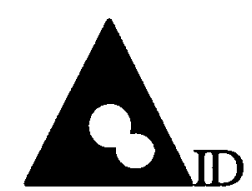

circle (left shape) circle (right shape) same-shape (left shape, right shape)

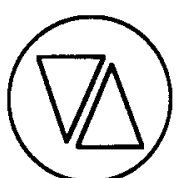

white (left triangle)

white (right triangle)

same-color (left triangle, right triangle)

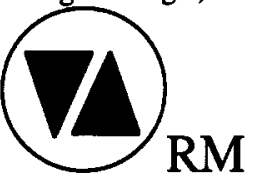

black (left triangle)

black (right triangle) same-color (left triangle, right triangle)

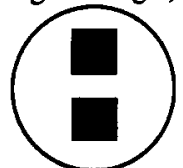

square (upper shape)

square (lower shape) same-shape (upper shape, lower shape)

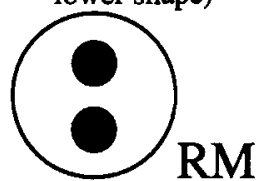

circle (upper shape)

circle (lower shape) same-shape (upper shape, lower shape)

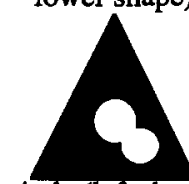

circle (left shape)

circle (right shape) same-shape (left shape,

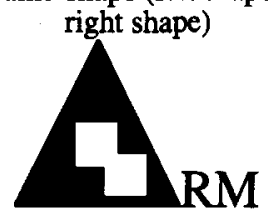

square (left shape) square (right shape) same-shape (left shape, right shape)

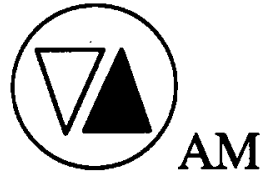

white (left triangle)

black (right triangle) different-color (left triangle, right triangle)

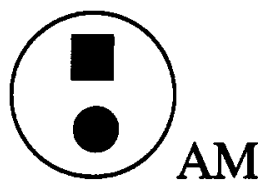

square (upper shape) circle (lower shape) different-shape (upper shape, lower shape)

Figure 3. Stimuli used in Experiment 1. The objects labeled ID are identical to the test object; the objects labeled $R M$ have a relational match with the test object; the objects labeled $A M$ have an attributional match with the test object.

same- and different-shade inducers. They found that inducing circles that were a different shade from the test circle produced the same magnitude illusion as inducing circles that were the same shade.

What is responsible for these failures to replicate? We noticed that all of our stimuli, as well as the stimuli of
Jaeger and Grasso (1993), were identical with respect to the perimeter. Coren and Miller (1974) only manipulated the similarity of the perimeters, suggesting that the similarity of the perimeters might be the important factor in determining the magnitude of the Ebbinghaus illusion. Furthermore, there were differences in the perimeters of 
Table 1

Magnitude of the Ebbinghaus Illusion and Its Significance Over Zero for Each of the Conditions in Experiment 1

\begin{tabular}{ccc}
\hline Condition & Magnitude $(\mathrm{mm})$ & \multicolumn{1}{c}{ Significance } \\
\hline First Set & & \\
ID & 1.875 & $t(51)=3.038, p<.005$ \\
RM & 0.567 & $t(51)=1.007, p>.3$ \\
AM & 2.019 & $t(51)=4.812, p<.005$ \\
Second Set & & \\
ID & 1.875 & $t(51)=3.513, p<.001$ \\
RM & 1.413 & $t(51)=3.137, p<.01$ \\
AM & 1.433 & $t(51)=2.837, p<.01$ \\
Third Set & & \\
ID & 1.529 & $t(51)=3.062, p<.005$ \\
RM & 0.981 & $t(51)=2.091, p<.05$ \\
AM & 0.913 & $t(51)=1.467, p>.1$ \\
\hline
\end{tabular}

Note-ID, the inducing objects were identical to their test objects; RM, the inducing objects had a relational match with their test objects; AM, the inducing objects had an attributional match with their test objects.

Coren and Enns's (1993) stimuli; perhaps those differences produced a confounding factor in their study. The hypothesis that the similarity with respect to the perimeter is the relevant factor in predicting the magnitude of the Ebbinghaus illusion might be able to explain all of these data.

To test this hypothesis, we decided to pit rated similarity against similarity with respect to the perimeter. For this purpose, two types of inducing objects were used. The inducing objects of the first type, the rated-similar (RS) inducing objects, had different perimeters from the test objects. However, we designed their centers to be similar to the centers of the test objects. As a result, participants were expected to (and did) rate these objects as similar to the test objects. The second type of inducing objects, the perimeter similar (PS) inducing objects, had similar perimeters to the test objects. The centers of these inducing objects were very different from the centers of the test objects and participants should not (and did not) rate these inducing objects as similar to the test object. We then surrounded the test objects with RS inducing objects in one condition and PS inducing objects in another to see which type would yield larger size contrast.

\section{EXPERIMENT 2}

\section{Method}

Participants. The participants were randomly approached by the experimenter in O'Hare International Airport on the northwest side of Chicago. Fifty-one people agreed to be in the experiment. All 51 participants appeared to understand the tasks required of them. As in Experiment 1, the participants served voluntarily.

Materials. We designed the three sets of stimuli shown in Figure 4. The inducing objects on the right (RS inducing objects) were rated by participants to be more similar to the test object overall, but the objects on the left (PS inducing objects) were more similar to the test object with respect to the perimeter.

In order to get the participants to rate the similarity of the RS and PS inducing objects to the test object, we prepared a booklet in which the participants could make these judgments. There were three $8.5 \times 5.5$ in. pages in this booklet, one for each of the three sets of stimuli. On each page of the booklet, the test object was placed on the top in the middle of the page. Immediately below the test object were the RS and PS objects. To control for order effects, in half of the booklets, the RS object was on the left and labeled A, and the PS object was on the right and labeled B; in the other half

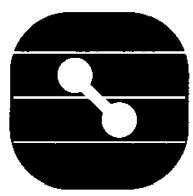

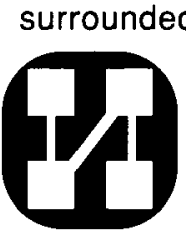

PS by big and small

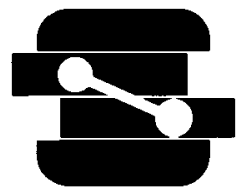

RS
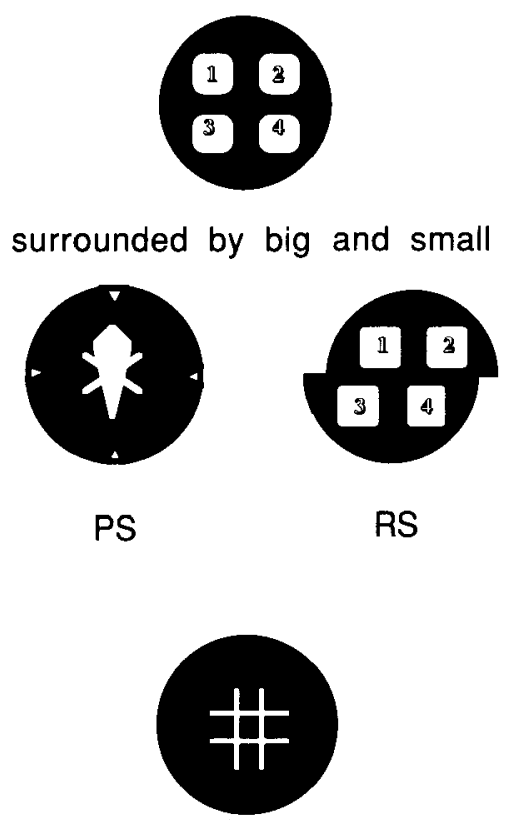

surrounded by big and small

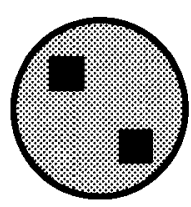

PS

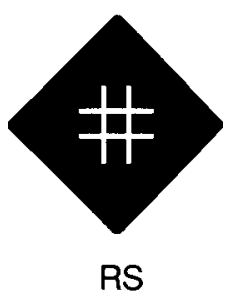

Figure 4. Stimuli used in Experiment 2. The perimeterically similar (PS) inducing objects are on the left. The rated similar (RS) inducing objects are on the right. 
of the booklets, the reverse was true. Below these objects, there were two 11 -point scales (from 0 to 10 ) on which the participants were to rate the similarity of the two inducing objects to the test object. Zero was labeled not similar, and 10 was labeled very similar.

With the exception of the four practice items at the beginning of the sequence, we designed each figure such that the maximum horizontal extent across the test object was $22 \mathrm{~mm}$. To create the RS and PS inducing objects, we started with designs that had the same dimensions as the test object and then transformed the RS objects into the shapes seen in Figure 4 . We then scaled the dimensions of the small inducing objects down to $5 / 11$ of their original size and scaled the dimensions of the big inducing objects up to $20 / 11$ of their original size. The same practice items with the same measurements as in Experiment 1 were used in Experiment 2. To control for any effects of the distance from the test object to the inducing objects, the PS inducing objects were exactly the same distance from the test object as the RS inducing objects within the same stimulus set. As in Experiment 1, all of these figures were drawn 1 per page on a computer and printed.

There were 28 items in all. Four of these items, the same ones as in Experiment 1, were practice items placed at the beginning of the sequence. To control for sequencing effects, the remaining 24 items were block randomized. These 24 items consisted of 12 test itemsthree sets of stimuli times two types of similarity relationships (PS and RS) times two (big and small inducers) - and 12 filler items. These last 24 items were numbered to allow the experimenter to keep track of them.

A booklet was prepared as in Experiment 1. The only difference between this booklet and the one in Experiment 1 was that there were only 24 numbered pages rather than 36 .

Procedure. The procedure here was identical to that of Experiment 1 with only one exception. Before beginning the Ebbinghaus task, the participants were given the booklet in which they were to make their similarity judgments and told to follow the instructions therein. After they had completed this task, they proceeded to perform the experiment in the same manner as described for Experiment 1 . The only remaining difference was that there were 28 trials rather than 40 trials.

\section{Results}

Looking first at the similarity ratings for each stimuli set, the RS inducing objects $(M \mathrm{~s}=6.18,7.18$, and 6.12) were, indeed, rated as significantly more similar to the test object than the PS inducing objects $(M \mathrm{~s}=2.27,1.92$, and 2.38 , respectively). These differences are highly reliable $[t \mathrm{~s}(50)=10.34,12.48$, and 9.6, respectively, $p \mathrm{~s}<.0001]$.

The magnitude of the Ebbinghaus illusion was measured in the same manner as in Experiment 1. As can be seen in Table 2, the mean magnitude of the illusion was greater than zero in all six cases, significantly greater than zero in five of those six. Of greatest relevance is the dissociation observed between illusion magnitude and rated similarity. A pooled ANOVA revealed that the PS inducing objects $(M=1.38)$ produced a significantly greater magnitude Ebbinghaus illusion than the RS inducing objects $(M=0.61)[F(1,52)=6.427, p<.02]$.

\section{Discussion}

These results demonstrate a dissociation between similarity judgments and the magnitude of the Ebbinghaus illusion and support the conclusion that the similarity of the perimeter is more important than rated similarity in
Table 2

Magnitude of the Ebbinghaus Illusion and Its Significance Over Zero for Each of the Conditions in Experiment 2

\begin{tabular}{ccc} 
Condition & Magnitude $(\mathrm{mm})$ & \multicolumn{1}{c}{ Significance } \\
\hline $\begin{array}{l}\text { First Set } \\
\text { RS }\end{array}$ & 0.814 & $t(50)=2.338, p<.05$ \\
PS & 0.971 & $t(50)=2.392, p<.05$ \\
Second Set & & \\
$\quad$ RS & 0.725 & $t(50)=2.022, p<.05$ \\
PS & 1.676 & $t(50)=4.491, p<.005$ \\
Third Set & & \\
RS & 0.304 & $t(50)=0.678, p>.5$ \\
PS & 1.5 & $t(50)=3.509, p<.005$
\end{tabular}

Note-RS, rated-similar inducing objects; PS, perimeter-similar inducing objects.

determining the magnitude of the Ebbinghaus illusion. Although perimeter similarity appears to be more important than center similarity, we do not know whether center similarity is irrelevant to the illusion. Furthermore, although these results confirm the hypothesis that having a different perimeter produces a decreased illusion magnitude relative to having a similar perimeter, we do not know whether illusion magnitude is a monotonic function of perimeter similarity, as suggested by our interpretation of Coren and Miller's (1974) results.

We decided to perform a $2 \times 2 \times 3$ (perimeter $\times$ middle $\times$ stimulus type) experiment to answer these questions and to replicate the dissociation between rated similarity and illusion magnitude. The test object was a circle for all stimulus types. For each of the three stimulus types, we surrounded these test circles with identicalperimeter (circle) inducers in one condition and differentperimeter inducers in another condition. For our different perimeter inducers, we used the Coren and Miller (1974) stimuli-a hexagon for one stimulus type, a triangle in another, and a random angular shape in the third-allowing us to test whether illusion magnitude is a monotonic function of perimeter similarity. The centers of all the inducers were either identical to or different from the center of the test object, allowing us to test whether there is any effect of the center on the magnitude of the illusion.

\section{EXPERIMENT 3}

\section{Method}

Participants. The participants were solicited randomly at $\mathrm{O}^{\prime} \mathrm{Hare}$ International Airport. One hundred twenty-one people agreed to be in the experiment. Of these 121 participants, 1 was dropped from analysis because of a failure to understand the task, leaving 120 participants. Of the 120 participants in the Ebbinghaus illusion portion of the experiment, 53 also completed the similarity judgment portion of the experiment. As in Experiments 1 and 2, all participants served voluntarily.

Materials. The three sets of stimuli shown in Figure 5 were used. The inducing objects on the far left $(\mathrm{P}+\mathrm{C}+)$ were identical to the test objects. The inducing objects next to them $(\mathrm{P}+\mathrm{C}-)$ had identical 


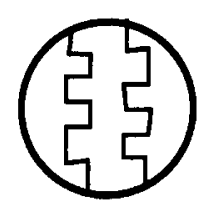

\section{surrounded by big and small}

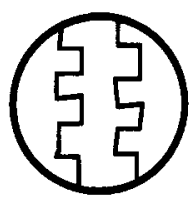

$\mathrm{P}+\mathrm{C}+$

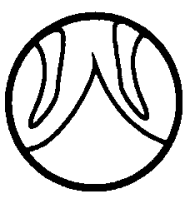

$\mathrm{P}+\mathrm{C}-$

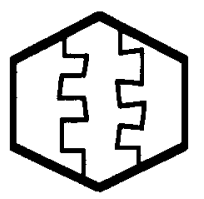

$\mathrm{P}-\mathrm{C}+$

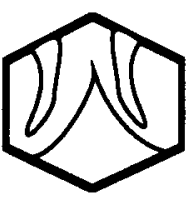

P-C-

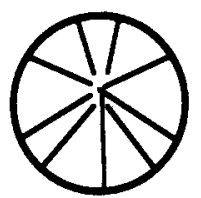

surrounded by big and small

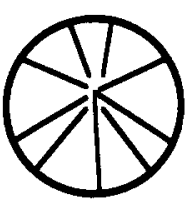

$\mathrm{P}+\mathrm{C}+$

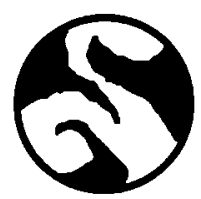

$\mathrm{P}+\mathrm{C}-$

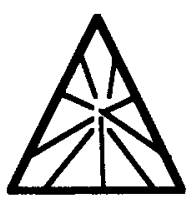

$\mathrm{P}-\mathrm{C}+$

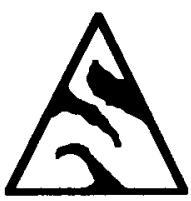

P-C-

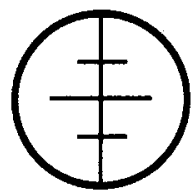

surrounded by big and small

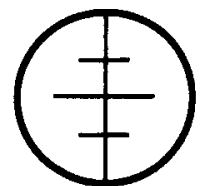

$\mathrm{P}+\mathrm{C}+$

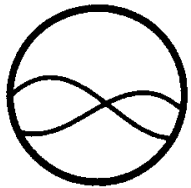

$\mathrm{P}+\mathrm{C}-$

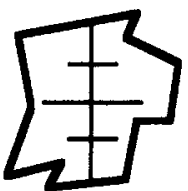

$\mathrm{P}-\mathrm{C}+$

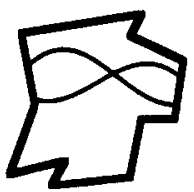

P-C-

Figure 5. Stimuli used in Experiment 3. The inducing objects on the left have an identical perimeter to the test object. The inducing objects on the right have different perimeters. These perimeters were inspired by Coren and Miller (1974). We also manipulated whether the centers were identical or different.

perimeters to the test objects. However, their centers were different. The objects just right of center $(\mathrm{P}-\mathrm{C}+)$ had different perimeters from the test objects. However, their centers were as identical to the centers of the test objects as was feasible given the constraint that the perimeters were different. The inducing objects on the far right $(\mathrm{P}-\mathrm{C}-)$ had different perimeters and different centers from the test objects.
We prepared a 2-page booklet for the similarity rating task. Each page was $8.5 \times 11$ in., with five similarity judgments. Among these 10 similarity judgments were 3 judgments of $\mathrm{P}-\mathrm{C}+$ inducing objects to their respective test objects, 3 judgments of $\mathrm{P}+\mathrm{C}-$ inducing objects to their respective test objects, and 4 filler judgments. These 10 judgments were arranged in a random order. At the top of 
the first page, the participants were instructed to rate the similarity of each pair. Each pair was presented with the test object on the left and the inducing object on the right. Immediately below each pair was a 7-point scale (from 1 to 7) on which the participants were to rate similarity: 1 was labeled not similar, and 7 was labeled very similar.

With the exception of the 4 practice items at the beginning of the sequence, we designed each figure such that the maximum horizontal extent across the test object was $22 \mathrm{~mm}$. All big inducing objects had a maximum horizontal extent of $44 \mathrm{~mm}$. All small inducing objects had a maximum horizontal extent of $11 \mathrm{~mm}$. Again, 4 practice items were used at the beginning of the experiment in order to give the participants practice with the method of reproduction. To control for any effects of distance from the test object to the inducing objects, all inducing objects were placed a constant $10 \mathrm{~mm}$ away from the test object. As in Experiments 1 and 2, all of these figures were drawn 1 per page on a computer and printed.

There were 28 items in all. Four of these items were practice items placed at the beginning of the sequence. To control for sequence effects, the remaining 24 items were block randomized. These 24 items consisted of three sets of stimuli types times two perimeter types (identical and different) times two center types (identical and different) times two (big and small inducers). These 24 items were numbered to allow the experimenter to keep track of them.

A booklet was prepared to allow the participants to judge the size of the test object using the method of reproduction, as in Experiments 1 and 2.

Procedure. The procedure here was identical to that of Experiment 2 . However, only 53 of the 120 participants made similarity judgments before continuing on to judge the width of the test object.

\section{Results}

Looking first at the similarity ratings for each stimuli set, the $\mathrm{P}-\mathrm{C}+$ inducing objects $(M \mathrm{~s}=3.98,3.73$, and 3.73 ) were, indeed, rated as significantly more similar to the test object than were the identical-perimeter different-

Table 3

Magnitude of the Ebbinghaus Illusion and Its Significance Over Zero for Each of the Conditions in Experiment 3

\begin{tabular}{|c|c|c|}
\hline Condition & Magnitude (mm) & Significance \\
\hline \multicolumn{3}{|l|}{ First Set } \\
\hline $\mathrm{P}+\mathrm{C}+$ & 0.988 & $t(119)=322, p<.01$ \\
\hline $\mathrm{P}+\mathrm{C}-$ & 1.008 & $t(119)=2.67, p<.01$ \\
\hline $\mathrm{P}-\mathrm{C}+$ & 0.696 & $t(119)=2.32, p<.025$ \\
\hline $\mathrm{P}-\mathrm{C}-$ & 0.779 & $t(119)=2.44, p<.025$ \\
\hline \multicolumn{3}{|l|}{ Second Set } \\
\hline $\mathrm{P}+\mathrm{C}+$ & 0.754 & $t(119)=2.65, p<.01$ \\
\hline $\mathrm{P}+\mathrm{C}-$ & 0.904 & $t(119)=3.06, p<.01$ \\
\hline $\mathrm{P}-\mathrm{C}+$ & 0.346 & $t(119)=1.09, p>.10$ \\
\hline $\mathrm{P}-\mathrm{C}-$ & 0.679 & $t(119)=1.98, p=.05$ \\
\hline \multicolumn{3}{|l|}{ Third Set } \\
\hline $\mathrm{P}+\mathrm{C}+$ & 0.688 & $t(119)=2.47, p<.05$ \\
\hline $\mathrm{P}+\mathrm{C}-$ & 0.604 & $t(119)=2.10, p<.05$ \\
\hline $\mathbf{P}-\mathrm{C}+$ & 0.275 & $t(119)=0.93, p>.10$ \\
\hline $\mathrm{P}-\mathrm{C}-$ & 0.029 & $t(119)=0.09, p>.10$ \\
\hline
\end{tabular}

Note- $\mathrm{P}+\mathrm{C}+$, the inducing objects were identical to the test objects; $\mathrm{P}+\mathrm{C}-$, the inducing objects had identical perimeters to the test objects, but their centers were different; $\mathrm{P}-\mathrm{C}+$, the inducing objects had different perimeters from the test objects, but their centers were as identical to the centers of the test objects as feasible given the constraint that the perimeters were different; $\mathrm{P}-\mathrm{C}-$, the inducing objects had different perimeters and different centers from the test objects. center inducing objects $(M \mathrm{~s}=2.27,2.19$, and 2.34 , respectively) $[t \mathrm{~s}(51)=7.96,6.87$, and 7.28 , respectively, $p \mathrm{~s}<.001]$.

The magnitude of the Ebbinghaus illusion was measured in the same manner as in Experiments 1 and 2. As can be seen in Table 3, the mean magnitude of the illusion was greater than zero in all 12 cases, reliably greater than zero in 9 of those 12 .

Furthermore, there was a significant effect of perimeter similarity on the magnitude of the illusion. The identicalperimeter inducing objects $(M=0.824)$ produced a significantly greater magnitude Ebbinghaus illusion than did the different-perimeter inducing objects $(M=0.467)$ $[F(1,119)=4.326, p<.05]$. A trend analysis shows a marginally reliable monotonic trend for the differentperimeter inducing objects $[F(1,119)=3.283, p<.08]$, such that the hexagons $(M=0.738)$ produced the greatest magnitude illusion, followed by the triangles $(M=$ $0.513)$, followed by the random angular objects $(M=$ 0.152 ). However, the identical-center inducing objects $(M=0.624)$ did not produce a greater magnitude Ebbinghaus illusion than did the different-center inducing objects $(M=0.667)[F(1,119)=0.056, p>.10]$.

\section{Discussion}

These results replicate the dissociation between magnitude of the illusion and rated similarity seen in Experiment 2 . In addition, we failed to find any effect of center similarity. Finally, there was a marginally reliable monotonic trend between perimeter similarity and illusion magnitude.

\section{GENERAL DISCUSSION}

Our initial goal was to see whether conceptual information plays a role in the Ebbinghaus illusion, as suggested by Coren and his colleagues. If so, then the magnitude of this illusion could be used effectively as an indirect measure of similarity. In Experiment 1, we tried to replicate the findings of Goldstone et al. (1991) using the magnitude of the Ebbinghaus illusion as the dependent variable. Not only did we fail to replicate their findings using this new method but also we found no evidence of similarity effects at all.

In Experiment 2, we asked whether the relevant factor in predicting the magnitude of the Ebbinghaus illusion might be the similarity of the perimeters. We set up a contrast between rated similarity and the similarity with respect to the perimeter by varying the center element. We found that illusion magnitude was a function of similarity of the perimeters and not rated similarity.

In Experiment 3, we failed to find any evidence of an effect of center similarity on the magnitude of the illusion. We also looked to see whether the magnitude of the illusion is a monotonic function of perimeter similarity, as suggested by Coren and Miller's (1974) stimuli. We 
found evidence to support this hypothesis. Finally, we replicated the dissociation between rated similarity and illusion magnitude.

Similarities and differences with respect to the perimeters might provide the most parsimonious explanation of the findings of Coren and his colleagues (Coren \& Enns, 1993; Coren \& Miller, 1974). Coren and Miller's experiment can be seen as a test of perimeter similarity. Their findings, therefore, support our hypothesis. Furthermore, this explanation could also account for Coren and Enns's results. Perimeter similarity covaried with conceptual similarity in their first and second experiments. As a result, the findings of these experiments are consistent with our hypothesis.

It is slightly more difficult to reconcile our findings with the results of Coren and Enns's (1993) third experiment in which the same figure was a magician wearing a hat from one orientation and a rabbit coming out of a hat from the other orientation. Since the same figure was used in both conditions, it would seem, at first glance, that the perimeters were identical in both conditions. Why, then, should there be a significant difference in the magnitude of size contrast between the two conditions? We believe that the change in orientation may be a critical factor in this experiment. A number of studies have shown that changing the orientation of some shapes changes their perceived shape (e.g., Humphreys, 1983). Coren and Enns tried to control for this change in orientation by showing that a change in orientation of a simpler, more schematized stimulus did not produce the same pattern of results. However, in simplifying and schematizing these stimuli, they changed a number of the shapes, including taking away the whiskers at the sides and changing the tie/ears from a curved figure to a triangular figure. Humphreys has shown that, although changes in orientation have an effect on the perceived shape of some shapes, they do not have such an effect on the perceived shape of others. A plausible explanation of the results of Coren and Enns's third and fourth experiments could be that the change in orientation caused a change in perceived shape of one stimulus (the magician/rabbit figure), but not of the other stimulus (the simplified, schematized figure).

The fact that we now have a dissociation between similarity judgments and the magnitude of the Ebbinghaus illusion makes it very unlikely that the illusion will ever serve as a reliable, implicit measure of general similarity or conceptual development. ${ }^{1}$ On the other hand, the results of our Experiment 3 and the results of Coren and Miller (1974) suggest that there is a monotonic relationship between the similarity of the perimeter and the magnitude of the Ebbinghaus illusion. Unless future research discovers a dissociation between the similarity of the perimeters and the magnitude of the Ebbinghaus illusion, this possibility leaves a potentially fertile ground for future research.

Why should the visual system care about the perimeters of objects? Estimating size requires participants to judge the distance from one side of the object to the other side of the object (i.e., the distance between two points on the perimeter). Information regarding perimeters, therefore, is important for size estimation.

The perimeter is also a global feature (Navon, 1977). One reviewer of this paper suggested the possibility that similarity with respect to global features, rather than the perimeter per se, is the most important factor in determining the magnitude of the Ebbinghaus illusion. This suggestion raises some interesting questions regarding what counts as an object. What would happen, for example, if we asked participants to judge the size of inner features rather than the size of the entire object? Would we find size contrast effects on these inner features? If global features are the driving force behind the similarity effects of the Ebbinghaus illusion, then one would expect to find no size contrast effects on these inner features. On the other hand, if similarity of contours determines which figures will be used as reference objects, we might find size contrast effects on these features. Furthermore, if features within the inducing objects have similar perimeters to the perimeter of the test object, then we might find that these inner features induce size contrast. These inner features would not be global features in the Navon sense. Such a finding would leave us with an interesting question regarding what counts as an object, since the inner features in such a case would effectively be acting as objects in themselves.

In our Experiments 2 and 3, we found an effect of perimeter similarity on the magnitude of the Ebbinghaus illusion. However, one might question why similarity should play any role at all in the magnitude of the illusion. Coren and Enns (1993) argued that, in order to judge size, it is desirable to take into account the conceptual similarity of the surrounding objects to the test object. We find their arguments compelling. However, if estimating size required one to determine category membership first, then the process of size estimation would be very expensive in terms of both computational resources and reaction time. Furthermore, such an algorithm would leave recognition processes without size information at their disposal. We postulate that the visual system has developed a more efficient approach - an approach that uses features that are diagnostic of, rather than isomorphic with, conceptual categories. The similarity of the perimeters or silhouettes is one such diagnostic feature, and there may be others.

\section{REFERENCES}

CoRen, S., \& EnNs, J. T. (1993). Size contrast as a function of conceptual similarity between test and inducers. Perception \& Psychophysics, 54, 579-588.

COREN, S., \& Girgus, J. S. (1972). A comparison of five methods of illusion measurement. Behavior Research Methods \& Instrumentation, 4, 240-244.

Coren, S., \& Miller, J. (1974). Size contrast as a function of figural similarity. Perception \& Psychophysics, 16, 355-357.

Goldstone, R. L., Medin, D. L., \& Gentner, D. (1991). Relations, at- 
tributes, and the non-independence of features in similarity judgments. Cognitive Psychology, 23, 222-262.

Humphreys, G. W. (1983). Reference frames and shape perception. Cognitive Psychology, 15, 151-196.

JaEgER, T., \& Grasso, K. (1993). Contour lightness and separation effects in the Ebbinghaus illusion. Perceptual \& Motor Skills, 76, 255-258.

Navon, D. (1977). Forest before trees: The precedence of global features in visual perception. Cognitive Psychology, 9, 353-383.

WeINTRAuB, D. J. (1979). Ebbinghaus illusion: Context, contour, and age influence the judged size of a circle amidst circles. Journal of Experimental Psychology: Human Perception \& Performance, 5, 353-364.

ZaNuTTINI, L. (1996). Figural and semantic factors in change in the Ebbinghaus illusion across four age groups of children. Perceptual \& Motor Skills, 82, 15-18.

\section{NOTE}

1. Zanuttini (1996), inspired by Coren and Enns (1993), argued for an effect of conceptual development on the magnitude of the Ebbinghaus illusion. However, there were differences in the perimeters of Zanuttini's stimuli. Furthermore, Weintraub (1979) found similar developmental effects using simple circles and contours. Perhaps the effects of perimeter similarity on the magnitude of the Ebbinghaus illusion also change with development.

(Manuscript received December 18, 1996; revision accepted for publication November 30, 1997.) 